\title{
Finite Element Modelling of Cracks as Acoustic Emission Sources
}

\author{
Markus G. R. Sause • Stefan Richler
}

Received: 21 August 2014 / Accepted: 16 January 2015 / Published online: 3 February 2015

(C) The Author(s) 2015. This article is published with open access at Springerlink.com

\begin{abstract}
This paper presents results for a new acoustic emission crack source model based on a finite element modelling approach which calculates the dynamic displacement field during crack formation. The specimen modelled is statically loaded until conditions for crack growth as defined by a failure criterion are fulfilled. Subsequently, crack growth is modelled by local degradation of the material stiffness utilizing a cohesive zone element approach. The displacements due to crack growth generate the acoustic emission signal and allow detailed examination of the principles of acoustic emission sources operation. Subsequent to crack growth signal propagation is modeled. The signal propagation is modeled superimposed on the static displacement field. The presented model comprises a multi-scale and multi-physics approach to consider the signal propagation from source to sensor, the piezoelectric conversion of the elastic wave to an electric signal and the interaction to the acquisition electronics. Validation of the modeling approach is done by investigating the acoustic emission signals of micromechanical experiments. Using a specifically developed load stage, carbon fiber filament failure and matrix cracking can be prepared as model sources. A comparison of the experimental signals to the modeled signals shows good quantitative agreement in signal amplitude and frequency content. A comparison between the present modeling work and analytical theories demonstrates the substantial differences not considered in previous modeling work of acoustic emission sources.
\end{abstract}

Keywords Finite element modeling - Acoustic emission . Fracture mechanics · Cohesive zone elements

M. G. R. Sause $(\varangle) \cdot$ S. Richler

Institute for Physics, Experimental Physics II, University of Augsburg, 86135 Augsburg, Germany

e-mail: markus.sause@physik.uni-augsburg.de

\section{Introduction}

The formation and propagation of cracks in solid media is a field of research that has been active for decades. Still, the theoretical description of the physics at the crack tip and the crack dynamics are active fields of research [1-4]. A phenomenon that is closely related to the crack dynamics is the generation of acoustic waves due to the crack motion due to the release of stored elastic energy. These acoustic waves propagate within the solid and can be detected at the surface by suitable sensor systems. This method is known as acoustic emission analysis and has already proven its significance for structural health monitoring as well as its ability to improve material testing procedures [5]. Despite of the broad range of technical applications, only a small amount of work has been performed recently to advance the understanding of the physical processes involved in the generation of acoustic emission.

In order to interpret the detected acoustic emission signals in terms of their relevance to material failure it is required to have concise knowledge of the underlying physics. The whole process of the acoustic emission technique can be categorized into three subsequent parts. The first part comprises the acoustic emission source, the second part considers the acoustic emission signal propagation from source to sensor and the third part consists of the acoustic emission signal detection.

In the past, various valuable attempts have been made to provide a theoretical description of acoustic emission sources. The source model concept used in most of the analytical approaches was derived from seismology and is most of the time based on the work of Aki and Richards [6]. Here, source models are geometrically approximated as point sources, while the dynamic of the source is either approximated from iterative refinement of model parameters to fit 

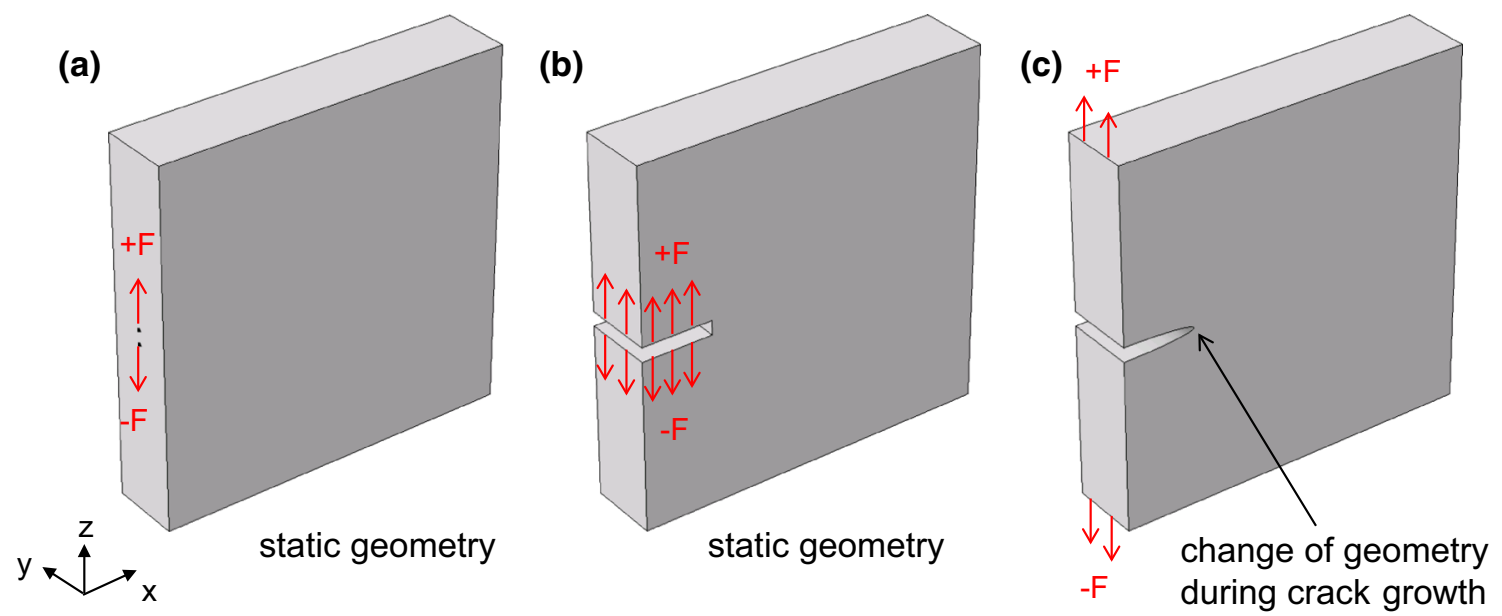

Fig. 1 Different types of acoustic emission source model descriptions used in literature employing point sources (a) or extended sources (b) in conjunction with analytic source functions. New source model description presented herein using dynamic changes of the source geometry based on fracture mechanics $(\mathbf{c})$

experimental data or is based on assumptions on the source dynamics derived from structural mechanics. Various stepfunction descriptions exist, which are used to describe the 3-dimensional spatial displacement of the crack surface during crack formation [7-11]. In particular, the rise-time of the initial crack surface displacement is an essential parameter to model the crack surface motion [12]. However, there are no reports in literature of successful measurements of risetimes of real acoustic emission sources, e.g. due to crack formation in materials. Instead the rise-time is typically estimated based on the elastic properties of the bulk material. This type of source modeling has been successfully applied to many cases, and the basic concept has been used within the generalized theory of acoustic emission by Ono and Ohtsu $[8,13]$, the work of Scruby [14] and numerous other analytical descriptions $[7,9,15,16]$.

In recent years it has become convenient to use numerical methods to model acoustic emission sources. In this field, Prosser, Hamstad and Gary applied finite element modeling to simulate acoustic emission sources based on body forces acting as a point source in a solid $[10,17]$. Hora and Cervena investigated the difference between nodal sources, line sources and cylindrical sources to build geometrically more representative acoustic emission sources [18]. At the same time, we proposed a finite element approach using an acoustic emission source model taking into account the geometry of a crack and the inhomogeneous elastic properties in the vicinity of the acoustic emission source [11].

Based on these investigations we can categorize the different modelling strategies to describe acoustic emission sources of crack propagation as shown in Fig. 1. The first type of source models considers point-like sources explicitly defining the source dynamics utilizing analytical source functions (cf. Fig. 1a). As second type we can interpret those attempts that have been made to incorporate more accurate source geometries, while the modeled crack dynamics are still based on analytical source functions (cf. Fig. 1b). The third type uses accurate artificial source geometries and does not need an analytical source function to generate acoustic emission. Instead, this type of source model is capable to generate the crack dynamics based on experimentally accessible parameters and fracture mechanics laws.

Currently all source models proposed in literature are of type one or type two, since they all require the definition of an explicit source function. Therefore, no details of the dynamics arising from the crack formation process and the subsequent crack surface motion are predicted or considered by those models.

From a mathematical modeling and simulation point of view, there are two main challenges in providing a numerically based acoustic emission source model of the third type. The first challenge consists of the different scales involved in the problem (crack length of the order of microns versus signal wavelength of the order of millimeters to centimeters) and the proper scale bridging. Owing to the vastly different observations scales, a full multi-scale approach is thus necessary. The second challenge stems from the calculation of temporal and spatial evolution of the surfaces of the crack. This is a level of detail that is typically not studied in modeling approaches used to describe crack formation by means of cohesive zone elements, extended finite element methods or similar implementations.

In contrast to the source model, the theoretical description and numerical implementation of wave propagation is already well established $[10,11,16,17,19,20,27]$. However, it is important to consider the effects of attenuation, dispersion and propagation in guiding media to accurately capture the characteristics of the signal (e.g. frequency content). 
While analytical descriptions benefit from the low computational intensity [16] to describe wave propagation, for the numerical methods a main focus is the improvement of the calculation routines.

Another challenge to obtain acoustic emission signals to compare relative to experimental data is the description of the detection process. Here the detection process using piezoelectric sensors can have significant impact on the bandwidth of the detected signals and their relative frequency content. While some analytical approaches consider the sensors transfer function explicitly $[16,21]$, some attempts have also been made to model the response of piezoelectric sensors themselves [22]. We recently proposed a finite element approach to directly include the piezoelectric sensor in a modeling environment to also account for the interplay between the sensor and the material it is attached on [23].

In the present work, we first present the source model description and establish its principle of operation. Subsequently we validate the source description by modeling of acoustic emission sources to correspond to micromechanical experiments. The experimental work is based on a micromechanics test stage, which was constructed to allow the preparation of fiber breakage and matrix cracking as acoustic emission sources. We then apply the new acoustic emission source model concept in combination with in-situ modeling of the signal propagation process and the detection process of a piezoelectric sensor. Comparison is made between the experimental results and the results from the different types of acoustic emission source simulation.

\section{Experimental Setup}

In the following, we present the experimental setup and the details of signal analysis as applied to the detected signals. As seen in Fig. 2, the core of the micromechanics test stage consists of an aluminum block with dimensions of $120 \mathrm{~mm}$ $\times 120 \mathrm{~mm} \times 39 \mathrm{~mm}$ (length $\times$ width $\times$ height). At the center of the top side of the aluminum block a pin with $2 \mathrm{~mm}$ diameter and $1 \mathrm{~mm}$ height was machined out of the block. At the opposite side of the block an acoustic emission sensor is positioned at the center. In order to ensure reproducible mounting conditions, we use a spring system to firmly press the sensor to the aluminum block.

For all cases investigated the first part of the preparation is to position a small droplet of RTM6 epoxy resin on top of the aluminum pin. To facilitate the positioning of the epoxy resin droplet a small depression was machined into the end of the aluminum pin. To prepare the micromechanical stage for generation of a matrix crack a tensile bar made from polyether ether ketone (PEEK) of $2 \mathrm{~mm}$ diameter and $80 \mathrm{~mm}$ length is used. The tensile bar is first moved into the liquid epoxy resin and then retracted to yield a tapered contour of 380 to $800 \mu \mathrm{m}$

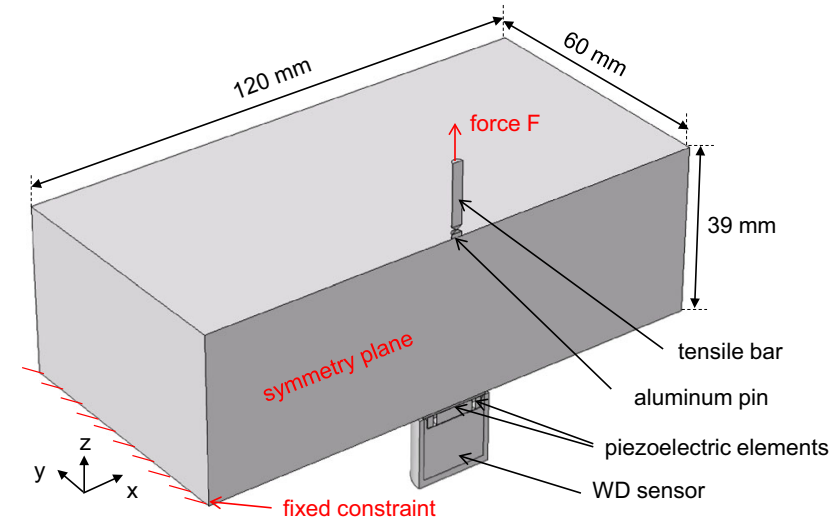

Fig. 2 3D-model of the experimental setup including cross-sectional view of acoustic emission sensor used

(see Fig. 3). Subsequently, curing of the liquid resin is carried out using heating foils attached to the aluminum block and an additional heating sleeve wrapped around a small cylinder covering the aluminum pin and parts of the tensile bar. We use a curing cycle comprising a heating rate of $2{ }^{\circ} \mathrm{C} / \mathrm{min}$ up to the curing temperature of $180^{\circ} \mathrm{C}$. The curing temperature is kept constant for 150 minutes with subsequent cooling to room temperature at a rate between 0.5 and $2{ }^{\circ} \mathrm{C} / \mathrm{min}$. Due to its low thermal conductivity the tensile bar made from PEEK minimizes the dissipative heat flux and therefore assures a constant temperature of the resin during curing. The tensile bar and the aluminum block are mounted in a universal test machine so that thermal expansion of the components and chemical shrinkage of the resin can be compensated by a closed loop force control. The test machine control adjusts the tensile bar position to assure zero force acting during curing, which is necessary to avoid excessive forces acting on the filament causing preliminary failure due to thermal expansion and cure shrinkage of the resin.

To prepare fiber breakage, we use a two-component epoxy to bond a HTA carbon fiber to the end of a flat-topped tensile bar made from PEEK. The fiber is then moved into the resin droplet using a micrometer stage and an optical microscope. The free fiber length was chosen to be between $350 \mu \mathrm{m}$ and $450 \mu \mathrm{m}$. The embed length was chosen larger than $100 \mu \mathrm{m}$ to reach fiber breakage before fiber pull-out occurs. After embedding, the resin droplet is cured in-situ.

After preparation of the test geometry, the universal test machine is used to apply a tensile force using a displacement controlled mode with velocities dependent on the selected failure mechanism. We choose a velocity of $20 \mu \mathrm{m} / \mathrm{min}$ for fiber breakage and $50 \mu \mathrm{m} / \mathrm{min}$ for matrix cracking. The failure was monitored by an optical microscope using a magnification factor of 100 . The images obtained after failure are shown in Fig. 3 for the two failure types. The respective acoustic emission signal is detected by a type WD piezoelectric sensor coupled by temperature stable Apiezon-L grease 

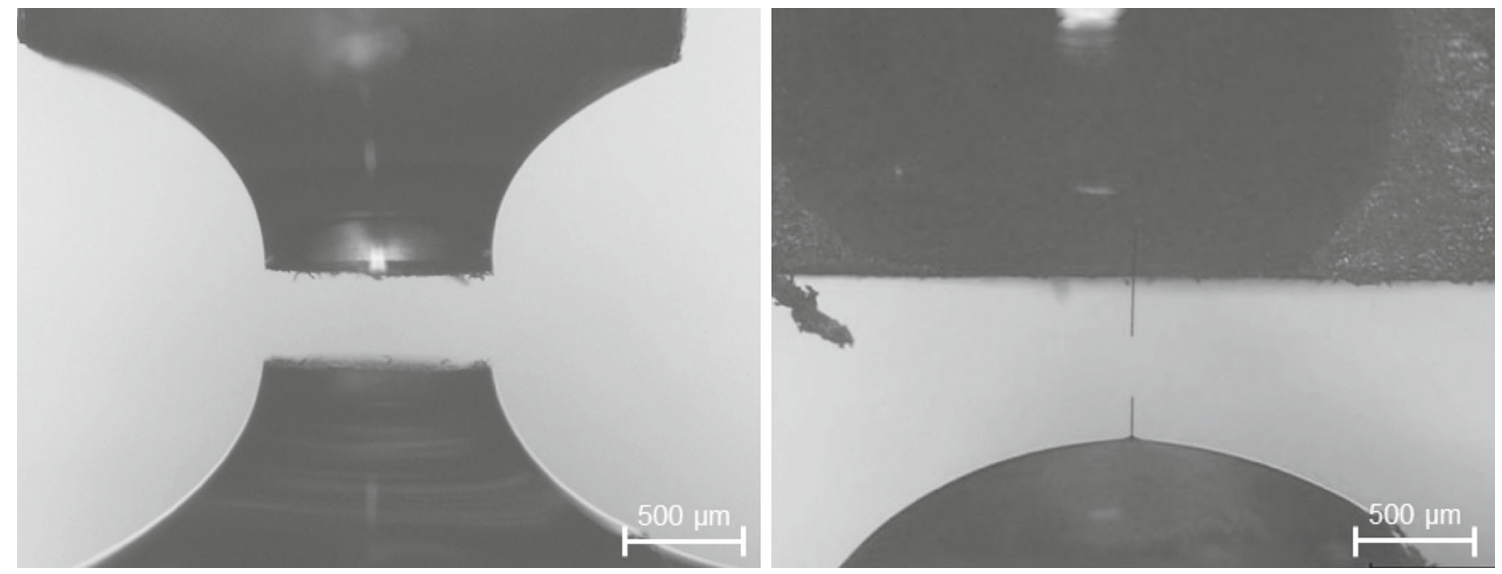

Fig. 3 Microscopy images of the failure mechanisms investigated: matrix cracking (left) and fiber breakage (right)

at the bottom of the aluminum block. The dimensions of the aluminum block allow an observation window of the primary acoustic emission signal of $18 \mu$ s free of reflections from the surfaces of the aluminum block. The detected acoustic emission signals are digitized by $40 \mathrm{MS} / \mathrm{s}$ using a bandpass range between $20 \mathrm{kHz}$ and $1 \mathrm{MHz}$. Triggering of the signals was carried out with $10 \mu$ s Peak-Definition-Time, $80 \mu$ s HitDefinition-Time and $300 \mu$ s Hit-Lockout-Time at a threshold level of $45 \mathrm{~dB}_{A E}$. The preamplification factor was chosen as $40 \mathrm{~dB}_{A E}$ for fiber breakage and as $20 \mathrm{~dB}_{A E}$ for matrix cracking.

\section{Model Description}

The model strategy uses the finite element method as implemented in the commercial software "COMSOL Multiphysics" and comprises a combination of multi-scale and multi-physics approaches. All calculations were carried out using the "structural mechanics module" as available in COMSOL version 4.4. All descriptions used in the following refer to this version of the software package.

The source model description proposed herein consists of three sequential modeling steps as schematically presented in Fig. 4. The first step is derived from classical structural mechanics. Suitable displacement boundary conditions are defined for the geometry considered to restrict some of the displacement components on one end (cf. Fig. 2). The other end is loaded by a force high enough to initiate fracture at the crack plane considered. If this force value is unknown, the implementation of a fracture criterion (e.g. fracture toughness, max. stress, etc.) to deduce the onset load for crack initiation is a straight forward procedure using a stationary solver sequence with incremental loading. If the external force is known from experiments, the measured force value can directly be used for the stationary solver. For the example shown in Fig. 4, the presence of the notch causes stress concentration at the tip of the notch, which will cause crack initiation at this point.

In the second step, the initial conditions for the displacement $\vec{u}$ and stress states $\vec{\sigma}$ are chosen to be identical to the static values $\vec{u}_{\text {static }}$ and $\vec{\sigma}_{\text {static }}$ as calculated in the previous step. Boundary conditions for restricted displacement components and external loads are kept identical to the previous step. In contrast to the previous step, now a transient calculation of the displacement field is performed. In addition, boundary conditions at the crack plane are chosen to allow for crack opening according to a fracture mechanics law. The duration of this transient calculation $t_{f r a c}$ is chosen to be sufficient until crack propagation has come to a rest. As seen from Fig. 4, the presence of the static displacement field causes crack propagation with an accompanying excitation of an acoustic emission wave. This spatial movement is seen best in the velocity field, since the static displacement dominates the displacement scale and therefore inhibits the identification of the very small displacements caused by the acoustic emission wave. A detailed discussion of the crack growth implementation is given in Sect. 3.1.

For the third step the initial conditions $(t=0)$ for the displacement, velocity and stress states of the last time value of the previous step $\left(t_{f r a c}\right)$ are used. Boundary conditions for restricted displacement components and external loads are kept identical to the first and second step. The boundary conditions applied at the crack plane are chosen to allow for independent movement of the new crack surfaces without allowing penetration of each other. This transient calculation is continued for a sufficient duration $t_{\text {end }}$ to allow for signal propagation in the test geometry as shown in Fig. 4.

\subsection{Implementation of Crack Propagation}

The present implementation of crack growth requires the definition of a fracture plane, similar to conventional cohesive zone modeling. In the example given in Fig. 4, the frac- 


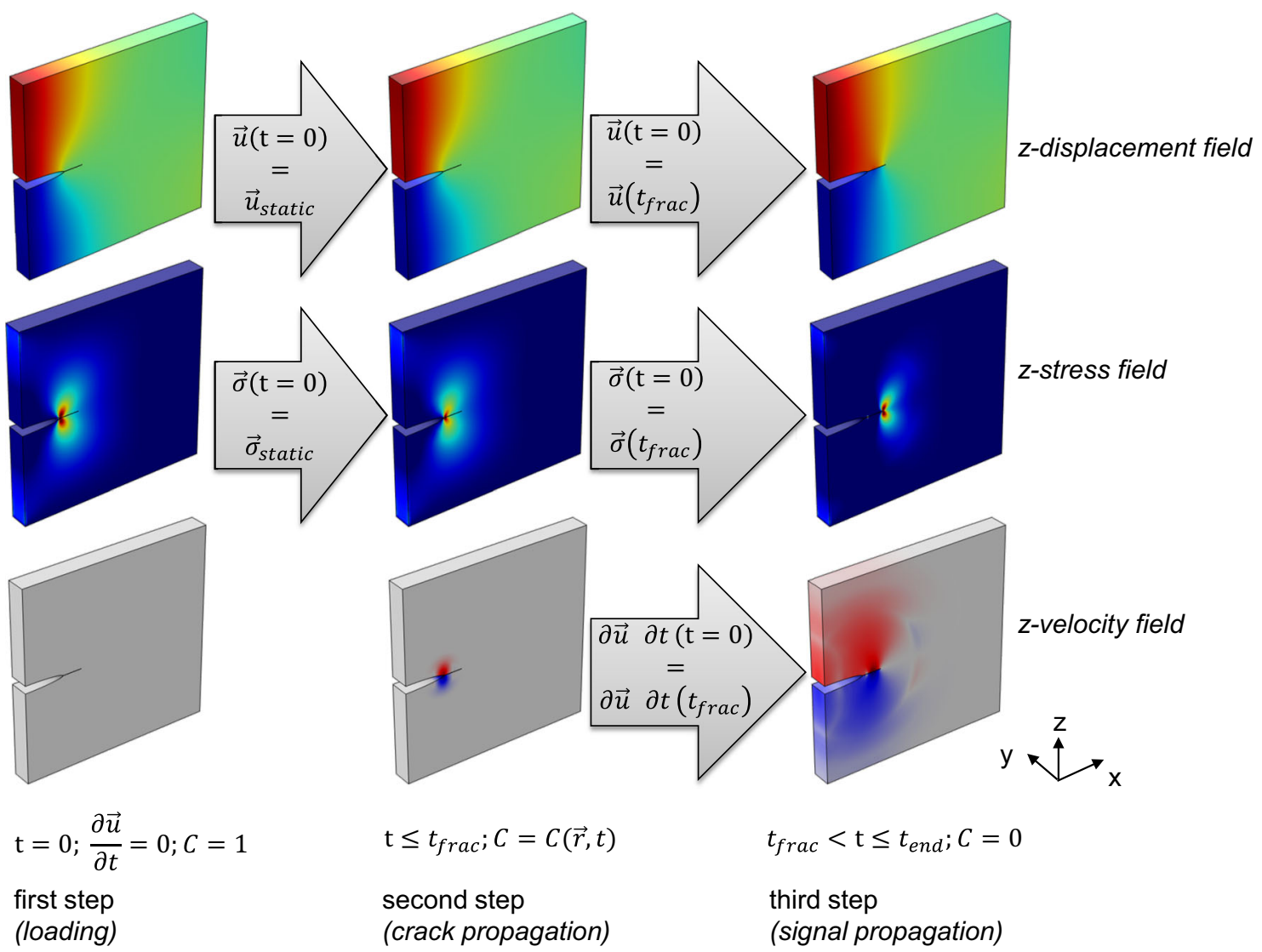

Fig. 4 Schematic of source model description using three subsequent modeling steps

ture plane chosen is the horizontal xy-plane located at the z-position of the notch tip. The extension of the fracture plane in $\mathrm{x}$-direction is chosen sufficiently large that the crack will not grow beyond the end of that plane. The boundary condition "thin elastic layer" as available in COMSOL 4.4 can then be defined for such an internal surface.

The stiffness vector $\vec{k}$ of this thin elastic layer is written in terms of the boundary coordinate system $\left(t_{1}, t_{2}, n\right)$, the Young's modulus $E$, the shear modulus $G$ and the Poisson's ratio $v$ as:

$\vec{k}=\left(\begin{array}{l}k_{t 1} \\ k_{t 2} \\ k_{n}\end{array}\right)$

$k_{t 1}=k_{t 2}=\frac{G}{t_{h}}$

$k_{n}=\frac{E(1-v)}{t_{h}(1+v)(1-2 v)}$

The parameter $t_{h}$ is an effective thickness associated with the thin elastic layer. The thickness value $t_{h}$ is chosen sufficiently small (i.e. $<1 \mathrm{~nm}$ ), so that the value of $\vec{k}$ has negligible influence on the overall compliance of the model.
To model crack growth, the stiffness vector is multiplied by a degradation function $C(\vec{r})$ evaluated as a function of the position on the fracture surface $\vec{r}$.

One example to define such a degradation function is the von Mises equivalent stress $\sigma_{v}$. For a general stress state this is written in terms of the normal stresses $\sigma_{i}$ and shear stresses $\tau_{i j}$ as:

$\sigma_{v}=\sqrt{\sigma_{x}^{2}+\sigma_{y}^{2}+\sigma_{z}^{2}-\sigma_{x} \sigma_{y}-\sigma_{x} \sigma_{z}-\sigma_{y} \sigma_{z}+3\left(\tau_{x y}^{2}+\tau_{x z}^{2}+\tau_{y z}^{2}\right)}$

Degradation of the stiffness vector $\vec{k}$ occurs if $\sigma_{v}$ exceeds the materials tensile strength $\sigma_{t}$.

For technical reasons, the Comsol environment also requires an additional ordinary differential equation to be defined on the fracture surface. This is to track the historic maximum value $\sigma_{\max }$ of $\sigma_{v}$. Therefore, the current implementation evaluates, whether the fracture condition is fulfilled in the present time step $i$ or was fulfilled in any previous time step.

Therefore the degradation function is written in terms of the maximum value of either $\sigma_{\max }$ or $\sigma_{v}$ : 
$C(\vec{r})=\left\{\begin{array}{l}1 \text { if } \max \left(\sigma_{\max }(\vec{r}), \sigma_{v}(\vec{r})\right)<\sigma_{t} \\ 0 \text { if } \max \left(\sigma_{\max }(\vec{r}), \sigma_{v}(\vec{r})\right) \geq \sigma_{t}\end{array}\right.$

For the brittle materials used in the present study, this simple description of material failure was found to be applicable. However, for materials involving larger amounts of plasticity prior to failure or significantly different interaction between normal stresses $\sigma_{i}$ and shear stresses $\tau_{i j}$ then assumed by Eq. (4) other formulations for Eq. (5) have to be used to capture the material behavior.

The advantage of the present description compared to other formulations for acoustic emission source models is the access to experimental parameters. In the proposed model, crack growth and acoustic emission is solely defined by the macroscopic loading condition and the failure criterion used. In particular, no explicit source function comprising internal forces or rise-times are necessary to initiate an acoustic emission signal.

\subsection{Discretization Settings and Material Properties}

We conducted convergence studies to set up the discretization levels used for the model. As the measure of comparison we use the displacement field values at the position of crack initiation (position specific for each model) and the acoustic emission voltage signal as computed by the modeled acoustic emission sensor. We use a refinement strategy for mesh and time resolution following [20]. Convergence is achieved, when signals of the refined model are within $97 \%$ coherence with the selected model. As measure of coherence we focus on the bandwidth between 0 and $10 \mathrm{MHz}$ for the evaluation of the displacement field at crack initiation and the bandwidth between 0 and $1 \mathrm{MHz}$ for the voltage signal of the acoustic emission sensor. This way of comparison follows the routine published in [20]. For the present model configuration seen in Fig. 2 we use a mesh resolution of a maximum edge length of $1.0 \mathrm{~mm}$ with several refinement steps when approaching the fracture plane and slight coarsening towards the edges of the aluminum block. The fracture plane itself is meshed with a maximum edge length of $0.4 \mu \mathrm{m}$. All elements are tetrahedral with quadratic geometry shape order.

For the time step we chose $0.01 \mathrm{~ns}$ during the process of crack growth for the carbon fiber breakage and $0.1 \mathrm{~ns}$ for the description of crack growth in the epoxy resin. This difference in convergent time step solutions is due to the vastly different sound velocities faced in these two material types. The duration $t_{\text {frac }}$ of this first transient calculation is carried out five times longer than the duration the crack needs to propagate through the material. This is to allow sufficient spreading of the high-frequency components before switching to a coarser time step in the subsequent step. This coarse time step was chosen as $10 \mathrm{~ns}$ and is used to compute sig- nal propagation within the test block and piezoelectric signal conversion for a total duration of $50 \mu \mathrm{s}$.

For the epoxy resin, the carbon fiber and the aluminum block we use the isotropic material properties as given in Table 1. The values for the epoxy resin and carbon fibers were obtained from in-house measurements following established standards. The values of the aluminum alloy were taken from the material supplier's datasheet. For the different materials included within the piezoelectric acoustic emission sensor, the required material properties are listed in Tables 1 and 2 using Voigt notation for the subset indices. The accuracy of the sensor model geometry and the according material properties was validated in detail in [24].

\subsection{Signal Detection and Post Processing}

In order to allow comparability to experimentally obtained signals the detection process by the acoustic emission sensor and the subsequent acquisition chain has to be taken into account. In the following we use a model of the WD sensor with parameters validated in [24] following the piezoelectric sensor modeling developed in [23]. The model uses the piezoelectric formulation used within the structural mechanics module of COMSOL. To consider the influence of the attached cable and the preamplifier we use the P-SPICE circuit simulation integrated in the ACDC module of COMSOL. The circuit outline follows the considerations in [23] comprising a low-pass given by a $10 \Omega$ resistor $90 \mathrm{pF}$ capacitor combination to model the sensor cable and a parallel series of a $10 \mathrm{k} \Omega$ resistor $15 \mathrm{pF}$ capacitor combination to model the preamplifier input properties. The acoustic emission signal is obtained as voltage across the $10 \mathrm{k} \Omega$ resistor (see [23] for detailed description and a circuit diagram). Although this explicit sensor modeling considers the multi-resonant behavior of the WD sensor, the band-pass characteristics of the preamplifier and the acquisition card are not accounted for in the simulation. Therefore the simulated voltage is subject to a subsequent band-pass filtering using a 6th order Butterworth high-pass of $100 \mathrm{kHz}$ frequency in combination with a 6th order Butterworth low-pass of $1,000 \mathrm{kHz}$ frequency.

\section{Results}

In the following we present the results of the source model computations. The first Sect. 4.1 deals with the temporal and spatial acoustic emission source activity. In Sect. 4.2 we compare the results of the modeling approach to experimental results for the setup shown in Fig. 2. Section 4.3 is dedicated to the comparison between the newly obtained results relative to acoustic emission source model strategies employing static geometries in conjunction with an analytic rise-time function (cf. Sect. 1). 
Table 1 Structural material properties used in the simulation process

\begin{tabular}{llll}
\hline Material & Density $\rho\left(\mathrm{kg} / \mathrm{m}^{3}\right)$ & Poisson-ratio & Elastic moduli (GPa) \\
\hline Epoxy resin (RTM6) & 1,140 & 0.38 & $\mathrm{E}=2.8$ \\
Carbon fiber (HTA) & 1,770 & 0.20 & $\mathrm{E}=240.0$ \\
Aluminum block (6063-T83) & 2,700 & 0.33 & $\mathrm{E}=69.0$ \\
Sensor case (stainless steel) & 7,970 & 0.29 & $\mathrm{E}=219$ \\
Backing material (Al-filled epoxy) & 2,700 & 0.40 & $\mathrm{E}=30.0$ \\
Acoustic insulation (PTFE) & 2,200 & 0.33 & $\mathrm{E}=0.4$ \\
Bonding (Ag-filled epoxy) & 1,700 & 0.45 & $\mathrm{E}=2.7$ \\
Wear plate (Al ${ }_{2} \mathrm{O}_{3}$ ) & 3,965 & 0.22 & $\mathrm{E}=400.0$ \\
Sensor elements (PZT-5A) & 7,750 & - & $\mathrm{C}_{11}=120.3$ \\
& & & $\mathrm{C}_{12}=75.2$ \\
& & & $\mathrm{C}_{13}=75.1$ \\
& & & $\mathrm{C}_{22}=120.3$ \\
& & & $\mathrm{C}_{23}=75.1$ \\
& & & $\mathrm{C}_{33}=110.9$ \\
& & & $\mathrm{C}_{44}=21.1$ \\
& & & $\mathrm{C}_{55}=21.1$ \\
& & & $\mathrm{C}_{66}=22.6$ \\
\hline
\end{tabular}

Table 2 Piezoelectric properties of PZT-5A

\begin{tabular}{lll}
\hline Material & $\begin{array}{l}\text { Coupling constants } \\
\left(\mathrm{C} / \mathrm{m}^{2}\right)\end{array}$ & $\begin{array}{l}\text { Relative } \\
\text { permittivity }\end{array}$ \\
\hline Sensor elements (PZT-5A) & $\mathrm{S}_{31}=-5.4$ & $\chi_{11}=919.1$ \\
& $\mathrm{~S}_{32}=-5.4$ & $\chi_{22}=919.1$ \\
& $\mathrm{~S}_{33}=15.8$ & $\chi_{33}=826.6$ \\
& $\mathrm{~S}_{24}=12.3$ & \\
& $\mathrm{~S}_{15}=12.3$ & \\
\hline
\end{tabular}

\subsection{Temporal and Spatial Acoustic Emission Source Activity}

For modeling of fiber breakage we use the experimentally obtained force value of $189 \mathrm{mN}$ as load in the first (stationary) step. As additional geometric modification to the description in Sect. 2 we use a small notch with $0.1 \mu$ m radius applied at one end of the fiber to produce a stress concentration at one edge of the specimen. This modification is motivated by fracture mechanics, which assumes the presence of a flaw in the material causing subsequent fracture. In reality it is likely, that failure will occur due to internal flaws at the position of highest stress concentration. The latter was observed to be at the position, where the carbon fiber is embedded in the resin. As seen by the images of the z-velocity field in Fig. 5, the crack initiates at the notch position and propagates through the carbon fiber at the designated fracture plane. The total duration for the crack length of $7 \mu \mathrm{m}$ is 1.2 ns, which approaches the crack tip velocity limit given by the Rayleigh velocity of $6652 \mathrm{~m} / \mathrm{s}$ calculated based on the approximation given by [25]. As consequence of the crack process a dynamic displacement field is generated. As previously noted in Sect. 3, this displacement field is hard to visualize due to the superimposed static displacement field. Therefore discussion is made with reference to the velocity field instead. Shown in Fig. 5 is the z-component of the velocity field. The formation of the velocity field follows the progress of the crack tip and the wave also propagates into the adjacent materials. The sound velocities of the carbon fiber and the resin part differ by one order of magnitude which causes substantially different distances of the wave front after $\mathrm{t}=1.4 \mathrm{~ns}$ in the two materials.

For the model of matrix cracking we use a notch with $1.0 \mu \mathrm{m}$ radius to initiate crack propagation on one edge of the tapered area of the resin similar to the fiber breakage model. As seen in Fig. 6, this also causes crack initiation at the designated site. Based on microscopic observations, the fracture plane is selected in the tapered area of the resin. Sometimes an inclination of the fracture planes and a natural roughness of the fracture surface were observed. However, to demonstrate some fundamental relationships it is advantageous to use this simple morphology of the fracture plane. In the stationary step, we use a force of $54 \mathrm{~N}$ as measured experimentally for the cross-section used. The resulting crack propagation in the resin takes $1.1 \mu \mathrm{s}$ for a crack length of 800 $\mu \mathrm{m}$. This again approaches the crack tip velocity defined by the Rayleigh velocity of $889 \mathrm{~m} / \mathrm{s}$ of the epoxy resin calcu- 


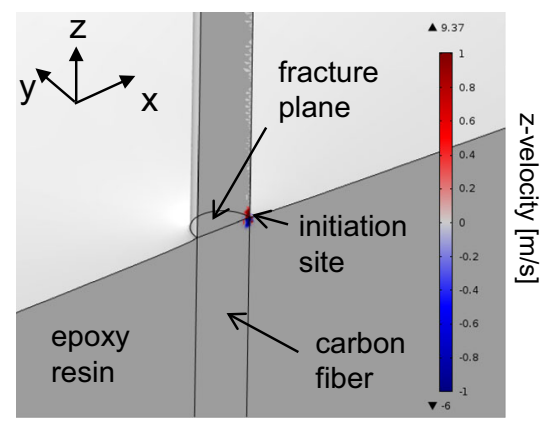

$\mathrm{t}=0.1 \mathrm{~ns}$

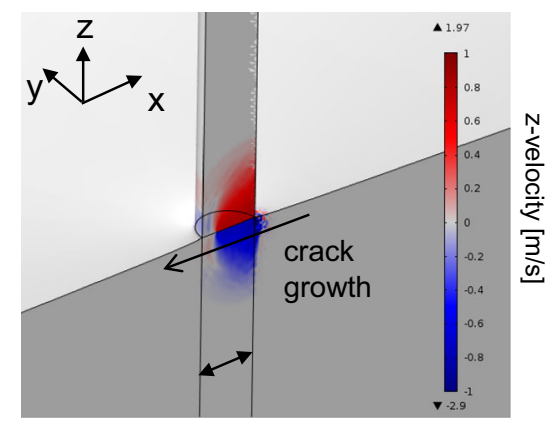

$\mathrm{t}=0.8 \mathrm{~ns}$

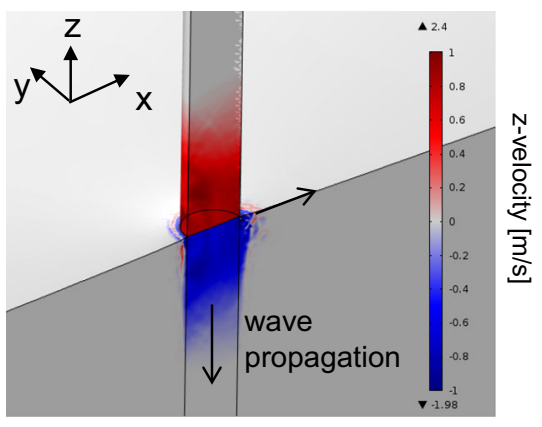

$\mathrm{t}=1.4 \mathrm{~ns}$

Fig. 5 Image stills of the acoustic emission source operation showing the $\mathrm{z}$-velocity field at $\mathrm{t}=0.1 \mathrm{~ns}, \mathrm{t}=0.8 \mathrm{~ns}$ and $\mathrm{t}=1.4 \mathrm{~ns}$ for fiber breakage

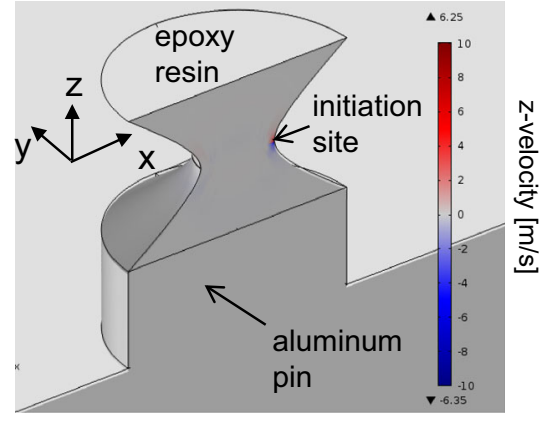

$\mathrm{t}=100 \mathrm{~ns}$

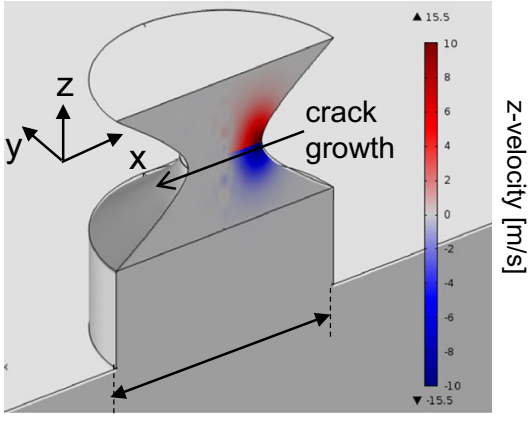

$t=450 \mathrm{~ns}$

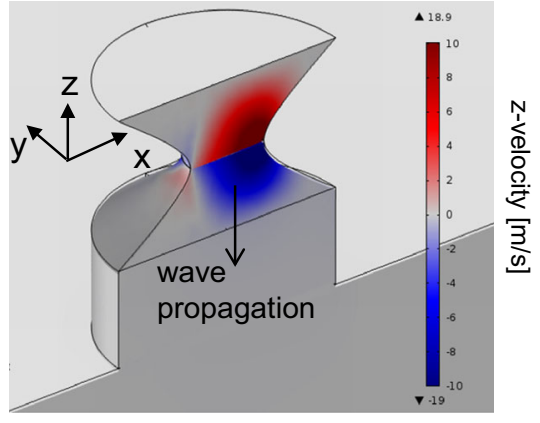

$\mathrm{t}=900 \mathrm{~ns}$

Fig. 6 Image stills of the acoustic emission source operation showing the $\mathrm{z}$-velocity field at $\mathrm{t}=100 \mathrm{~ns}, \mathrm{t}=450 \mathrm{~ns}$ and $\mathrm{t}=900 \mathrm{~ns}$ for matrix cracking

lated based on the approximation given by [25]. As consequence of the crack propagation, an acoustic wave propagates into the adjacent materials, which is subject to immediate interaction with the nearby boundaries, the newly formed crack surface and the different velocities of the adjacent materials.

In order to discuss this matrix source model relative to previous modeling concepts, a cross-section evaluation is performed using the cut-plane function in Comsol. Therefore we evaluate the average $\mathrm{z}$-displacement of the lower half of the fracture surface using a position slightly offset (shifted by $\mathrm{z}=-10^{-22} \mathrm{~m}$ ) to the initial position.

The respective evaluation of the z-displacement of the fracture surface of the fiber breakage model is shown in Fig. 7a, b. During the short duration of crack propagation $t_{\text {frac }}$, the z-displacement increases continuously, but does not settle at the moment of crack-through. Instead, the $\mathrm{z}$ displacement increases further until a maximum value is reached. Subsequently, the fracture surface starts to vibrate and settles at a new equilibrium position. This vibration of the fracture surface has been reported in previous modeling attempts $[11,24,27]$ and points out one important difference to the classically assumed source functions including only a step-function like behavior. The presence of these surface vibrations superimposed to a step-function rise of the fracture surface was also recently validated by means of electromagnetic emission measurements [28]. However, the present case considers fracture of a free fiber on the upper half, so no further boundary constraints act on the surface of the fiber. This situation will be slightly different in a fiber reinforced composite, where the bond of the fiber to the surrounding matrix will reduce the amount of vibration of the fiber surface.

The predictions of the maximum z-displacement $z_{\text {theory }}$ calculated according to the theory of Green [26] is marked as a dashed line in Fig. 7b. This is evaluated using the Young's modulus $E$ and Poisson ratio $v$, as well as the accumulated stress $\sigma$ and the crack propagation length $a$ :

$z_{\text {theory }}=\frac{4 \cdot(1-v)}{\pi \cdot E} \cdot \sigma \cdot a$

As can be seen from the comparison, for the case of fiber breakage, the analytical value is significantly lower than the result of the numerical model. This is attributed to various reasons. For a simple case following the assumptions of static extension of a flaw due to an external load as made by Green [26] the results between the numerical model and the analytical computation were found to be in $99 \%$ agreement. But as soon as dynamic displacements are taken into 

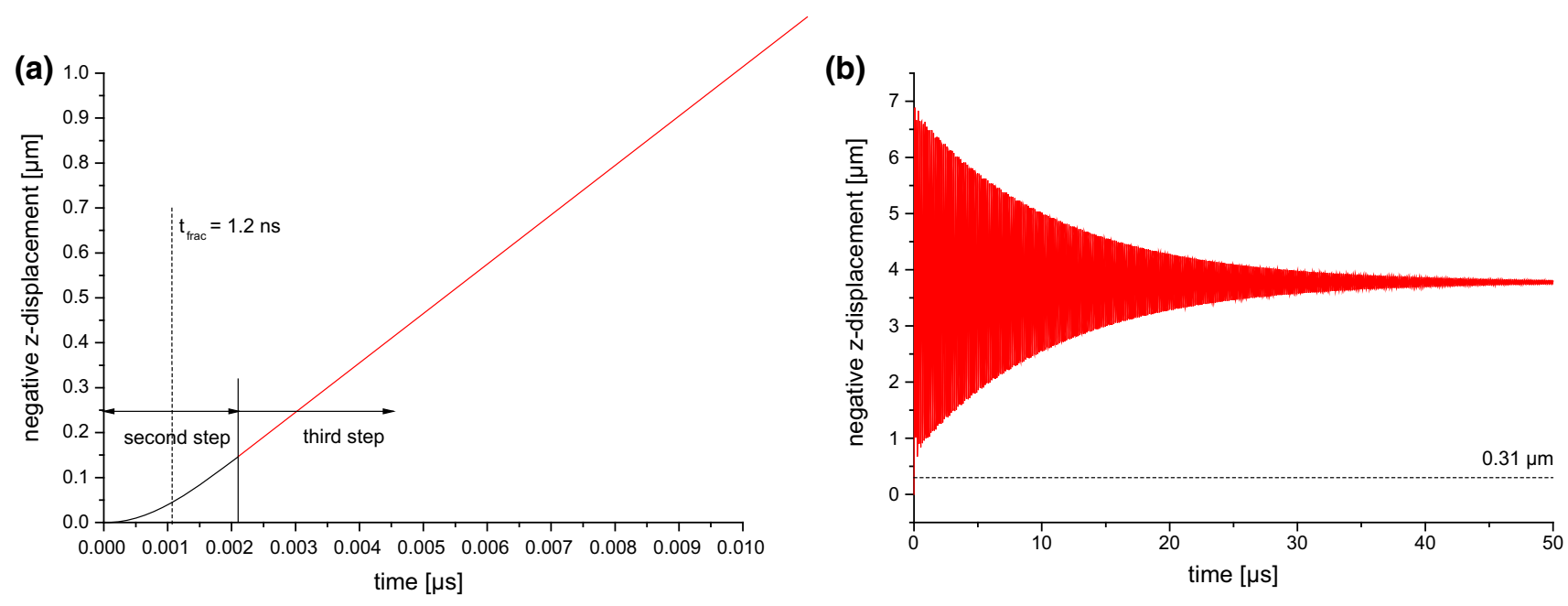

Fig. 7 Average z-displacement of the fracture surface for fiber breakage in the initial part (a) and for the full duration of the computation (b). For comparison the analytic solution of Greens theory is given as dashed line
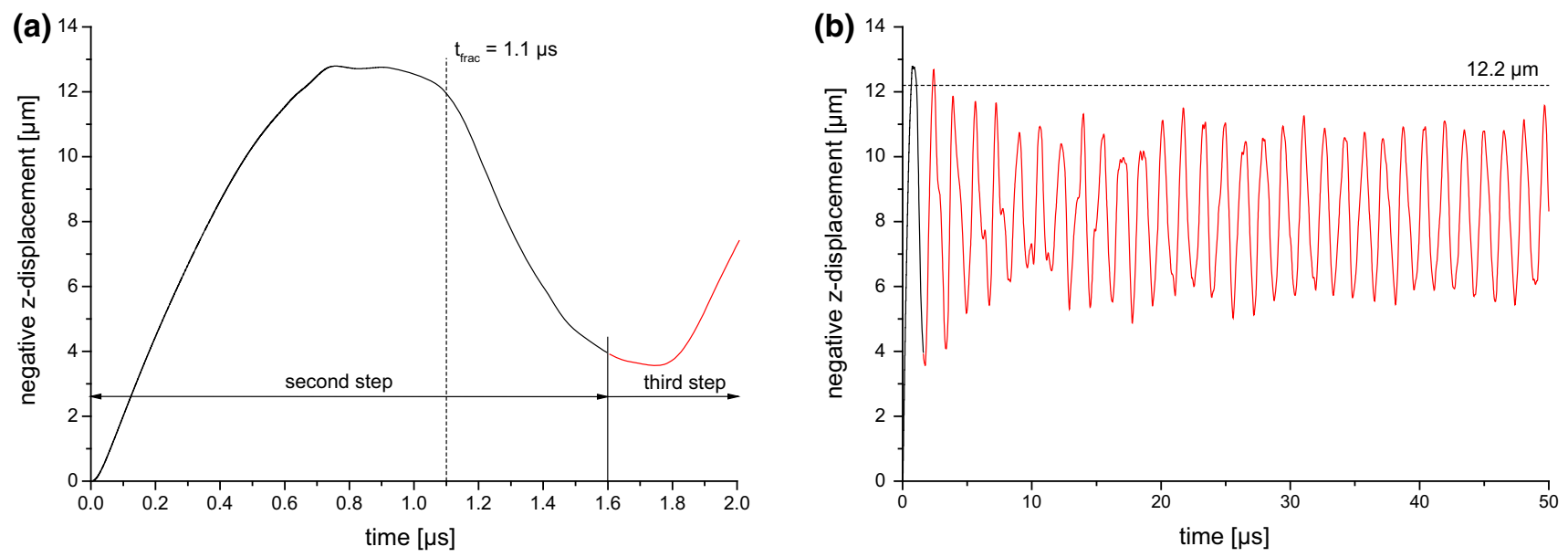

Fig. 8 Average z-displacement of the fracture surface for matrix cracking in the initial part (a) and for the full duration of the computation (b). For comparison the analytic solution of Greens theory is given as dashed line

account, the analytical prediction underestimates the strength of acoustic emission sources. For the present geometry it is hard to approximate the situation as a notched beam with infinite extension in one axis. Therefore, additional geometrical effects are likely, which also cause deviation of the source energy release. However, as will be demonstrated in Sect. 4.2, the computed source displacements turn into acoustic emission signals, which are in good agreement with the experimental signals.

In Fig. 8a, b the average z-displacement of the fracture surface of the matrix crack is shown. This was evaluated using a cut-plane analogous to the procedure for fiber breakage. Compared to the fiber breakage case there are several obvious differences. The initial rise of the signal is slower than for fiber breakage. This is owed to the different Rayleigh velocities limiting the speed of crack propagation and the different length of crack propagation faced in the two setups. More- over, the oscillation frequency is different and does not decay as fast as for fiber breakage. This is also attributed to the difference in Rayleigh wave velocity and the different geometry. Also, the maximum source displacement occurs before final crack-through. This is due to the averaging process of the $\mathrm{z}-$ displacement of the full fracture surface. In the beginning the newly forming fracture surface close to the initiation moves in the negative z-direction. After a certain time the movement of this part of the fracture surface settles and starts to move in the positive z-direction. The latter movement is already present before the final crack-through and therefore contributes to the averaging process. The prediction given by the theory of Green [26] is still lower than the peak value, but is systematically above the average z-displacement levels after $\mathrm{t}=3 \mu \mathrm{s}$. This difference is again owed to the geometric differences between the assumptions made in [26] and the present situation seen in Fig. 6. 

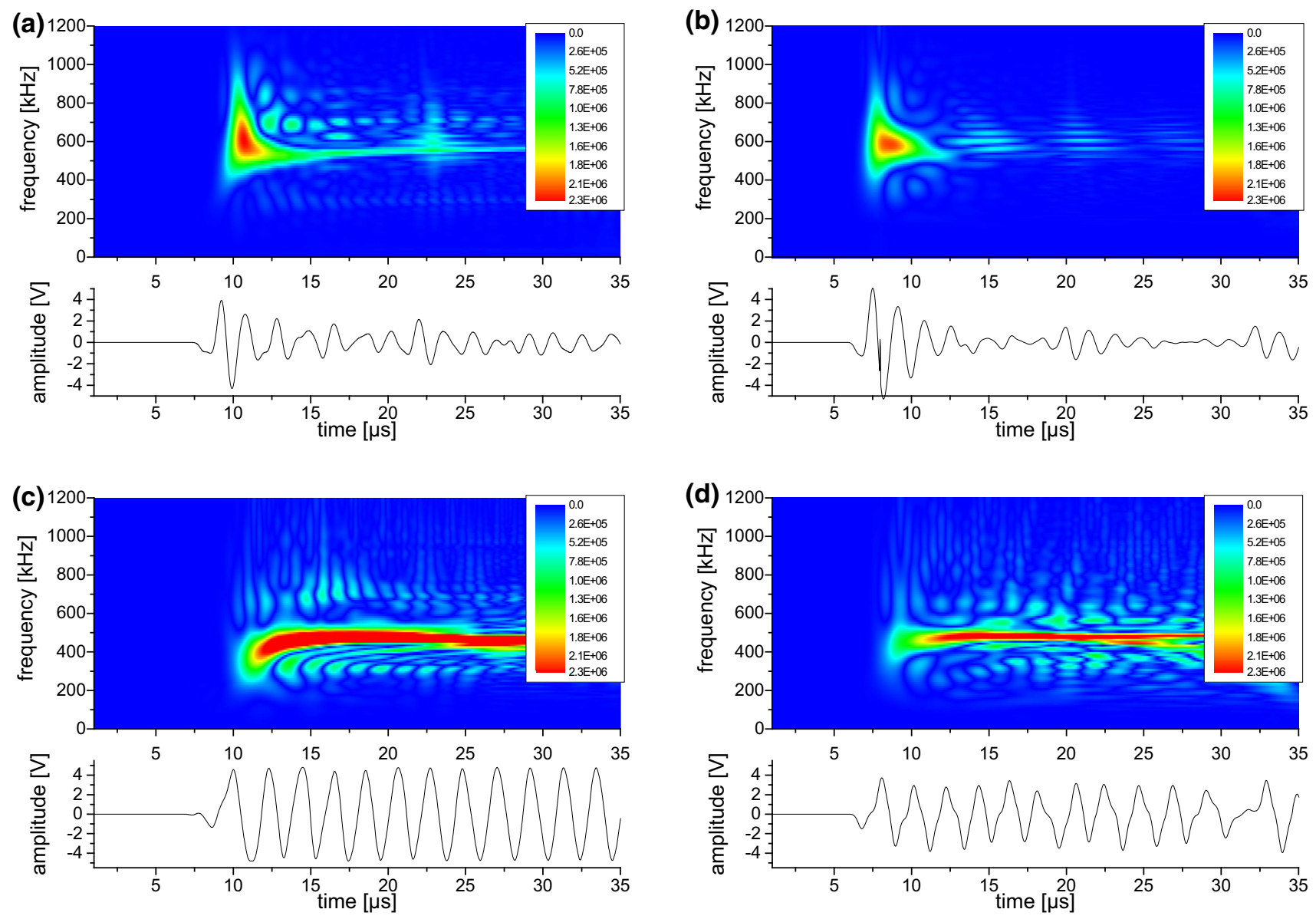

Fig. 9 Comparison between simulated $(\mathbf{a}, \mathbf{c})$ and experimental $(\mathbf{b}, \mathbf{d})$ results of fiber breakage and matrix cracking, respectively

\subsection{Comparison to Experimental Results}

In this section comparison is made between the results obtained from the numerical modeling and the corresponding experimental signals. As noted in Sect. 3, the modeled signals are the result from a full 3D computation including piezoelectric conversion within the sensor model application of a P-Spice circuit simulation and subsequent band-pass filtering. After amplification of the modeled signals by 20 $\mathrm{dB}_{A E}$ or $40 \mathrm{~dB}_{A E}$ (see Sect. 2) this allows for direct comparison of the acoustic emission signal amplitudes in the voltage scale.

For the case of fiber breakage, the comparison is found in Fig. 9a, b. As seen from the voltage scale and the timefrequency signature given in the Choi-Williams distribution there is very good agreement between the modeled and the experimental signal. In particular, the signal amplitudes show almost identical peak values and the echoes of the initial pulse are adequately captured. Therefore, we assume the source function and intensity as reported in Sect. 4.1 to be valid for the fiber breakage case. Moreover, this also indicates, that the von Mises failure criterion is applicable for the present case.

Also for the matrix cracking case shown in Fig. 9c, $d$ there is very good agreement in the voltage scales and the timefrequency signature shown in the Choi-Williams distribution. Slight differences arise in the modeled signal after $\mathrm{t}=10 \mu \mathrm{s}$. This is due to the repetitive approach-retract cycles of the newly formed fracture surface. In the modeling part, those fracture planes are partially restricted in their relative motion due to the selected symmetry plane. Therefore their dominant movement direction is in the $\mathrm{z}$-direction. In the experimental part, the fracture surface might experience additional sliding and torsional motions as well as additional interlocking of rough surface parts. This may account for the smoother appearance of the spikes in the experimental signals. However, the signal amplitudes are in good agreement, which also indicates the validity of the source movement reported in Sect. 4.1 and the applicability of the von Mises failure criterion for the present modeling work. 

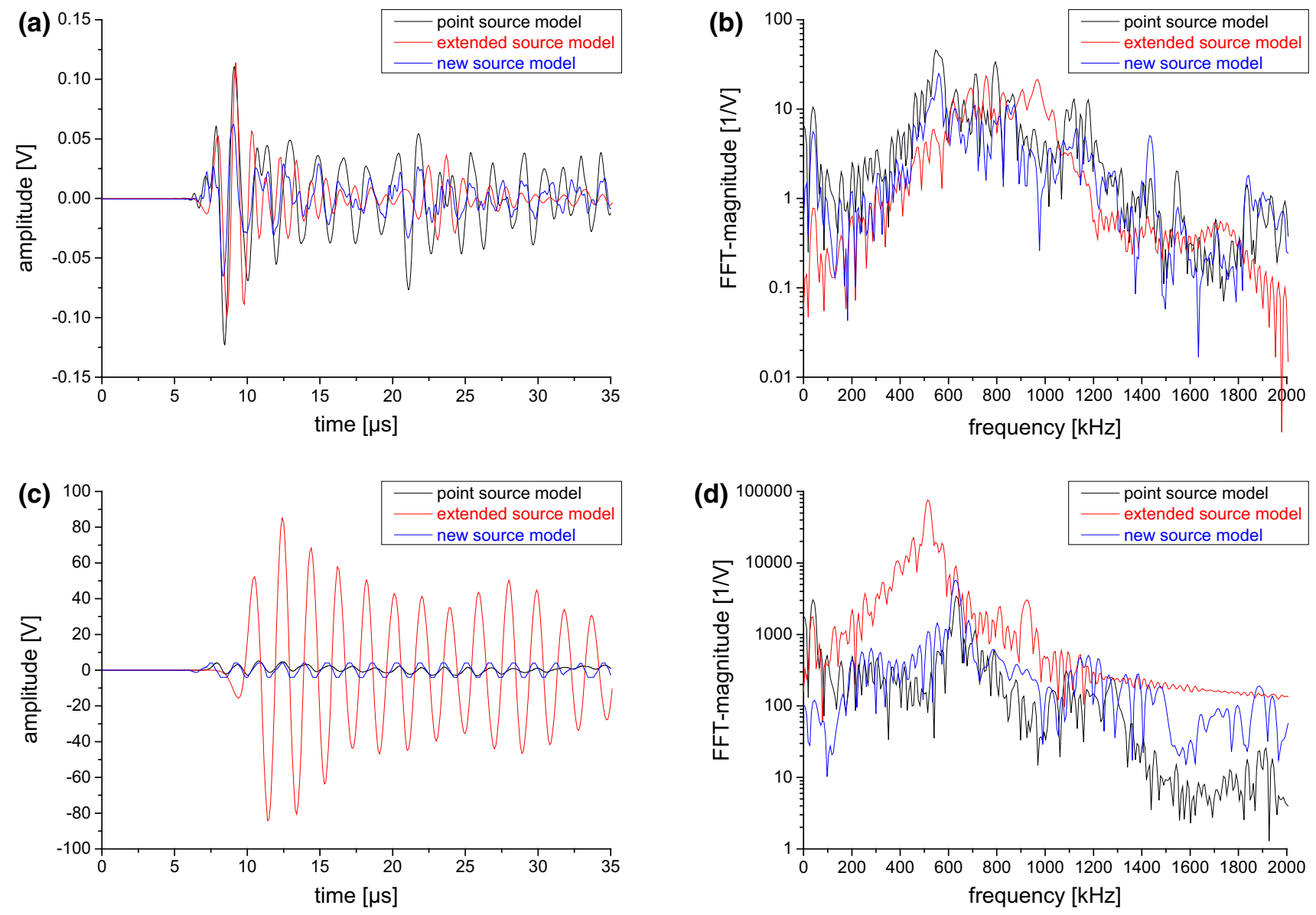

Fig. 10 Comparison of computation results for three different source model descriptions for fiber breakage case (a,b) and matrix cracking (c,d)

\subsection{Comparison Between Different Source Modeling Strategies}

After comparing the results of the newly proposed source model to experimental results, the aim of this section is to discuss the relevance relative to previous source modeling concepts.

Therefore, the model setup shown in Fig. 2 is taken as an example and signals are calculated in this identical geometry using the same sensor model, but using three different source model concepts.

The first source model concept will be referred to as point source model. This model uses the implementation of an acoustic emission source as internal point couples applying a cosine bell force-time function with the experimentally measured force values of $189 \mathrm{mN}$ and $54 \mathrm{~N}$ for fiber breakage and matrix cracking, respectively. As rise-time $0.1 \mu$ s is selected for the fiber breakage model and $1.0 \mu \mathrm{s}$ is used for the matrix cracking model since these were calculated to be the duration until the maximum z-displacement was reached for fiber breakage and matrix cracking, respectively (cf. Figs. 7b, 8b). A simple dipole representation of $1 \mu \mathrm{m}$ axis length directed along the z-axis is chosen. The dipole is positioned at the center of the fiber and the tapered area of the resin, respectively.

As second model concept, the same prescribed forcetime source functions are used. However, the active area is extended to the full fracture surface. Therefore the full fiber cross-section and the full resin cross-section are subject to the cosine bell step function. This model will be referred to as extended model. The third model concept uses the newly proposed implementation described in the sections above and will therefore be referred to as new source model.

In Fig. 9a, b a comparison is made between the unfiltered results of the three source types for modeling fiber breakage. This was chosen to discuss the differences of the three descriptions in the highest possible bandwidth (i.e. not limited by the experimentally used range). As seen in Fig. 9a for the fiber breakage case, all three models yield comparable source amplitudes. Also, the signals frequency content and shape are still in reasonable agreement as seen in Fig. 9b. Considering the geometrical arrangement as seen in Fig. 5, this is not unexpected. Although the fiber has a certain geometrical extension, the excited wavelengths are of the same 
or larger dimension. Therefore, despite of the geometrical complexity of the source it seems sufficient to be described by a single dipole source operating at this position.

For the case of matrix cracking shown in Fig. 9c this seems to be different. Here, the point source models result in amplitudes, which are of the same order of magnitude as the new source model. In contrast, the extended source model overshoots this range by a factor of four. But the signal arrivals, the amplitudes and the frequency content (cf. Fig. 9d) do not show a close match for the three cases. This is readily explained by the dimension of the source relative to the wave lengths involved. As seen by the spreading of the wave field in Fig. 6, the size of the source is two orders of magnitude larger than for the fiber breakage case. Hence, the wave length of the initially emitted wave starts to interfere with the surrounding boundaries and causes interference with the wave still being emitted by the source. Therefore the spatial position and sequence of excitation does play an important role, which is not adequately captured by a point source model or the extend source model. Hence, the newly proposed source model is expected to yield a more realistic description of the displacement field caused by the crack propagation (Fig. 10).

\section{Conclusion}

We presented a new approach to model acoustic emission sources. In contrast to previous source model descriptions, the proposed model does not require definition of a force-time curve as source function. Moreover, no assumptions regarding directivity of source couples, their relative intensities or positioning is required. The present source model operates solely based on accessible experimental parameters such as external loads, a constitutive equation for the material and a fracture mechanics based failure criterion. The latter initiates and stops crack propagation using a cohesive zone element modeling approach. This enables an improved representation of acoustic emission sources and avoids additional assumptions on source strengths or source rise-times not accessible by experimental means.

The acoustic emission signals generated by the present source model description have been validated against experimental signals obtained from micro-mechanical experiments. In these experiments the failure mode is easily observable using microscopy imaging and the external load is straight-forward to measure. The model also comprises a full 3D representation of the according propagation medium (large aluminum block) and a model of a commercially used acoustic emission sensor. The latter implies a piezoelectric conversion process in conjunction with a subsequent P-SPICE circuit simulation to account for the impact of the preamplifier. Simulated and experimental acoustic emission signals were found to show very good agreement. It was demonstrated that previous source model descriptions, such as point couples or prescribed static geometries cannot account for the dynamic processes around the source once the geometrical dimensions of the source approaches the wavelength of the generated signals. However, careful revision is required for the applied failure criterion and constitutive equations, if large plastic deformation is expected prior to failure or other interaction between normal and shear stress components occurs.

As with all cohesive zone modeling approaches, the explicit definition of a fracture surface also requires some assumptions. However, for simple load cases, the position of the fracture surface is straightforward or readily deduced from microscopic observations after fracture. Also, inclusion of more complex fracture surfaces to account for further details of experimental fracture morphologies is straightforward in the approach presented.

Acknowledgments I would like to thank Marvin Hamstad and Malte Peter for the fruitful discussions on acoustic emission source modeling.

Open Access This article is distributed under the terms of the Creative Commons Attribution License which permits any use, distribution, and reproduction in any medium, provided the original author(s) and the source are credited.

\section{References}

1. Livne, A., Bouchbinder, E., Fineberg, J.: Breakdown of linear elastic fracture mechanics near the tip of a rapid crack. Phys. Rev. Lett. 101, 264-301 (2008)

2. Livne, A., Bouchbinder, E., Svetlizky, I., Fineberg, J.: The near-tip fields of fast cracks. Science 327, 1359-1363 (2010)

3. Buehler, M., Gao, H.: Dynamical fracture instabilities due to local hyperelasticity at crack tips. Nature 439, 307-310 (2006)

4. Fineberg, J., Gross, S.P., Marder, M., Swinney, H.L.: Instability in dynamic fracture. Phys. Rev. Lett. 67, 457-460 (1991)

5. Große, C., Ohtsu, M.: Acoustic Emission Testing in Engineering: Basics and Applications. Springer Verlag, Berlin (2008)

6. Aki, K., Richards, P.G.: Quantitative Seismology, Theory and Methods. University Science Books, Sausalito (1980)

7. Giordano, M., Calabro, A., Esposito, C., D'Amore, A., Nicolais, L.: An acoustic-emission characterization of the failure modes in polymer-composite materials. Compos. Sci. Technol. 58, 19231928 (1998)

8. Ohtsu, M., Ono, K.: The generalized theory and source representation of acoustic emission. J. Acoust. Emiss. 5, 124-133 (1986)

9. Lysak, M.: Development of the theory of acoustic emission by propagating cracks in terms of fracture mechanics. Eng. Fract. Mech. 55(3), 443-452 (1996)

10. Hamstad, M., O'Gallagher, A., Gary, J.: Modeling of buried acoustic emission monopole and dipole sources with a finite element technique. J. Acoust. Emiss. 17(3-4), 97-110 (1999)

11. Sause, M., Horn, S.: Simulation of acoustic emission in planar carbon fiber reinforced plastic specimens. J. Nondestruct. Eval. 29(2), 123-142 (2010)

12. Hamstad, M.: On lamb modes as a function of acoustic emission source rise time. J. Acoust. Emiss. 27, 114-136 (2009) 
13. Ohtsu, M., Ono, K.: A generalized theory of acoustic emission and Green's function in a half space. J. Acoust. Emiss. 3, 27-40 (1984)

14. Scruby, C.: Quantitative acoustic emission techniques. Nondestruct. Test. 8, 141-210 (1985)

15. Schubert, F.: Numerical Modeling of Acoustic Emission Sources and Wave Propagation in Concrete NDT.net 7, 9 (2002)

16. Wilcox, P., Lee, C., Scholey, J., Friswell, M., Wisnom, M., Drinkwater, B.: Progress towards a forward model of the complete acoustic emission process. Adv. Mater. Res. 13-14, 69-76 (2006)

17. Prosser, W., Hamstad, M., Gary, J., O'Gallagher, A.: Comparison of finite element and plate theory methods for modeling acoustic emission waveforms. J. Nondestruct. Eval. 18(3), 83-90 (1999)

18. Hamstad, M.A., O'Gallagher, A., Gary, J.: Modeling of buried acoustic emission monopole and dipole sources with a finite element technique. J. Acoust. Emiss. 17(3-4), 97-110 (1999)

19. Hora, P., Cervena, O.: Acoustic emission source modeling. Appl. Comput. Mech. 4, 25-36 (2010)

20. Rose, J.: Ultrasonic Waves in Solid Media. Cambridge University Press, Cambridge (1999)

21. Sause, M., Hamstad, M., Horn, S.: Finite element modeling of lamb wave propagation in anisotropic hybrid materials. Compos. B 53, 249-257 (2013)

22. McLaskey, G., Glaser, S.: Acoustic emission sensor calibration for absolute source measurements. J. Nondestruct. Eval. 31(2), 157$168(2012)$
23. Cervena, O., Hora, P.: Analysis of the conical piezoelectric acoustic emission transducer. Appl. Comput. Mech. 2, 13-24 (2008)

24. Sause, M., Hamstad, M., Horn, S.: Finite element modeling of conical acoustic emission sensors and corresponding experiments. Sensors Actuators A 184, 64-71 (2012)

25. Sause, M.: Identification of Failure Mechanisms in Hybrid Materials Utilizing Pattern Recognition Techniques Applied to Acoustic Emission Signals. mbv-Verlag, Berlin (2010)

26. Bergmann, L.: Der Ultraschall und seine Anwendung in Wissenschaft und Technik, p. 560. Hirzel-Verlag, Stuttgart (1954)

27. Green, A., Zerna, W.: Theoretical Elasticity. Oxford University Press, London, New York (1954)

28. Burks, B., Kumosa, M.: A modal acoustic emission signal classification scheme derived from finite element simulation. Int. J. Damage Mech. 23(1), 43-62 (2013)

29. Gade, S., Weiss, U., Peter, M., Sause, M.: Relation of electromagnetic emission and crack dynamics in epoxy resin materials. J. Nondestruct. Eval. 33, 711-723 (2014) 